ORIGINAL STUDY

\title{
Clinical application of laryngeal electromyography - a point of view
}

\author{
Georgiana Pasu \\ ENT\&HNS Department, "Sfanta Maria" Hospital, Bucharest, Romania \\ "Carol Davila” University of Medicine and Pharmacy, Bucharest, Romania \\ CESITO Centre, "Sfanta Maria" Hospital, Bucharest, Romania
}

\begin{abstract}
In recent years, the clinical importance of laryngeal electromyography (LEMG) has been recognized. This method of investigation proved itself to be a valuable and indispensable tool regarding voice disorder management and requires multidisciplinary approach.

In this article, we focus on the clinical usefulness of LEMG to assess inferior and superior motor neuron integrity by recording action potentials generated by muscle fibers of the larynx in the new field known as neurolaryngology. To facilitate the clinical application of this electrophysiologic test, we present a detailed description of LEMG techniques, which were applied for simultaneous bilateral recordings of the thyroarytenoid, cricothyroid and posterior cricoarytenoid muscles. Also, we analysed and measured the following electrophysiological parameters: 1) insertional activity; 2) spontaneous activity; 3) recruitment; 4) morphology of waveform.

From a practical point of view, in our country, LEMG performance is still not a routine procedure in ENT and Phoniatric Departments. The difficulty of practical use of LEMG in Romania consists in technical limitations and it depends on the physician's experience regarding the examination technique and also the interpretation of electrophysiological results.

Our current clinical experience shows that the use of LEMG contributes especially to the diagnosis, prognosis and treatment of patients with neurolaryngological disorders.
\end{abstract}

KEYWORDS: electromyography, neurolaryngology, larynx, recurrential paralysis

\section{INTRODUCTION}

Laryngeal electromyography (LEMG) is one of the most important developments of neurolaryngology in the diagnosis and treatment of voice disorders. Laryngeal electromyography, along with laboratory investigation (videostroboscopy, fiberoptic laringoscopy, neurotoxicity tests, chest x-ray, CT, MRI), is considered an extension of the ENT clinical examination and the electromyography abnormalities records should be interpreted within the clinical context.

Neurological, inflammatory and traumatic injuries of the superior laryngeal nerve are diagnosed through EMG of the cricothyroid muscle. The electrical activity of the branches of the recurrent laryngeal nerve is eval- uated through bilateral electromyographic examination of the thyroarytenoid muscle (TA), the lateral cricoarytenoid (LCA) muscle, the posterior cricoarytenoid (PCA) and the interarytenoid muscle. EMG of the intrinsic muscles innervated by the recurrent laryngeal nerve involves the examiner's experience and a detailed knowledge of the anatomy of the larynx ${ }^{1}$.

This diagnosis method has been recognized since 1944 by Weddel and there is an important research regarding its application. Even if it has been performed since the $1950 \mathrm{~s}^{2}$, its practical clinical importance has been emphasized only since the last three decades of the twentieth century $y^{2,3}$.

The variations of the technique implemented over time, the variability patterns of the study, the different 
models of needle electrodes used and the different approaches of analysing the records lead to different results among physicians and researchers ${ }^{4}$. In 2010, a working group of neurolaryngologists was created to reach a consensus regarding the guidelines for LEMG performance: indications, technique, interpretation of records and clinical application ${ }^{5}$.

In 2012, a valid scientific article stated that the laryngeal electromyography is a good assessment in predicting the prognosis of recovery in patients diagnosed with vocal fold paresis and that it can help identify patients suitable for surgery ${ }^{6}$. It was also observed that the results of LEMG recordings repeatedly obtained on the same patient with neurologic diseases that affect the larynx are hardly reproducible ${ }^{7}$.

LEMG has been recently introduce in the ENT\&HNS Department of "Sfanta Maria" Hospital (October 2015). Since then, we perform electromyographic recordings in all patients diagnosed with vocal fold immobility using standardised techniques of this objective investigation and we try to correlate our results with those obtained by other more experienced physicians ${ }^{7}$.

In our country, this objective assessment of the vocal fold pathology represents an area insufficiently studied because laryngologists did not have access to advanced electromyographers in their departments and many neurologists do not have enough training regarding laryngeal anatomy.

\section{INDICATIONS FOR LEMG}

LEMG is an important objective investigation in assessment of the vocal fold pathology, especially when it is performed 12-14 days after the onset of the vocal fold immobility. LEMG is represented as being the "gold standard" investigation method used in the diagnosis of vocal fold paralysis or paresis due to the recurrent laryngeal nerve and superior laryngeal nerve lesion.

There are different causes that can lead to vocal fold paresis: 1) malignant disease: lung cancer, oesophageal cancer and thyroid cancer; 2) surgical trauma: thyroid surgeries are the most common, mediastinal surgeries, oesophageal surgeries; 3) cervical trauma, head trauma; 4) inflammatory pathology: viral postherpetic neuritis; 5) systemic neurological and neuromuscular disorders: amyotrophic lateral sclerosis, Guillain-Barré, post-polio syndrome, Parkinson's, Myasthenia gravis; 6 ) idiopathic ${ }^{7,8}$.

It is particularly helpful for the differential diagnosis between vocal fold paralysis and arytenoid dislocation. It is also an important tool considering psychiatric patients (suspected hysterical dysphonia) ${ }^{7,8}$.

\section{PROCEDURE AND TECHNICAL CONSIDERATIONS}

\section{Requirements and preparations}

The protocol of LEMG requires first of all signing an informed consent form by the patient. Before starting this investigation, the technique protocol, including the complete equipment and the minimally invasive manoeuvres, has to be explained to all patients, in order to obtain a fairly and accurate electrodiagnostic. In order to achieve a good quality of EMG signals, the patient is required to actively participate and properly collaborate in this procedure; the discomfort will be minimised.

A detailed examination of the larynx, including a cervical CT scan or MRI, an indirect or fiberoptic laryngoscopy and/or videostroboscopy, is useful to detect altered anatomy of the head and neck, previous operations of the vocal cords, or acute infections - situations which can potentially hinder the electrode insertion ${ }^{8}$.

Generally, this procedure does not involve local anaesthesia, but if the patient has a high threshold of pain, local anaesthesia with lidocaine can be applied transorally or with a transcutaneous intraluminal injection. The trick that helps us performing an accurate EMG is marked by the absence of local anaesthetic $^{9}$. The European Laryngological Society states that the local anaesthetic drug will modify the electrical signals of the intrinsic muscles, influencing the results and distorting the analysis of the motor unit action potential (MUAP) ${ }^{8}$.

Relative contraindications of using LEMG are represented by: bleeding disorders caused by administration of warfarin or acetylsalicylic acid, coagulopathy and patients with pacemakers ${ }^{8}$.

If the patient is diagnosed with bilateral vocal cord paresis and she/he has tracheostomy, in order to place the needle electrode, it is usually necessary to remove the tracheotomy tube. During electrode insertion manoeuvre, the patient may experience shortness of breath. For maintaining the airway open during the examination, a nasal speculum may be used to dilate the orifice of the tracheostomy ${ }^{8}$.

\section{Equipment}

EMG amplifier. The acoustic analysis of the EMG signal is performed after connecting a speaker to an audio amplifier, which in turn it is also connected to a converter to provide computer-readable data. Performed EMG devices afford the automatic detection and the detailed analyses of the motor unit action potential (MUAP) ${ }^{8}$.

Electrode type. There are several types of needle electrodes that can be used while performing the LEMG: the monopolar needle, the bipolar concentric needle and single-fiber needle electrodes. 
For the transcutaneous approach of the laryngeal muscles, it is proposed to use the concentric needle electrodes which determine a uniform field for MUAP waveform analysis. The maximum length of the needle electrode should be $50 \mathrm{~mm}$ in order to penetrate even the posterior cricoarytenoid muscle (PCA) ${ }^{10}$ (Figure 1).

Transnasal flexible laryngoscopy is the investigation that can be done routinely before, during and after laryngeal electromyography, in order to highlight the endolarynx and the vocal cord mobility ${ }^{8}$. Using a fiberoptic scope, moving the needle within the thyroarytenoid muscle and observing the vocal fold movement, the position of the electrode can be identified ${ }^{8}$ (Figure 1).

\section{The insertion of the electrode}

The LEMG begins with the insertion of the needle into the thyroarytenoid muscle and the posterior cricoarytenoid muscle, assessing the recurrent laryngeal nerve (RLN) integrity. To examine the function of the superior laryngeal nerve (SLN) the needle should be inserted into the cricothyroid muscle (CT).

The first step in the transcutaneous approach is to identify the laryngeal landmarks in the cervical anterior midline: lower border of the thyroid and cricoid cartilage, cricothyroid membrane.

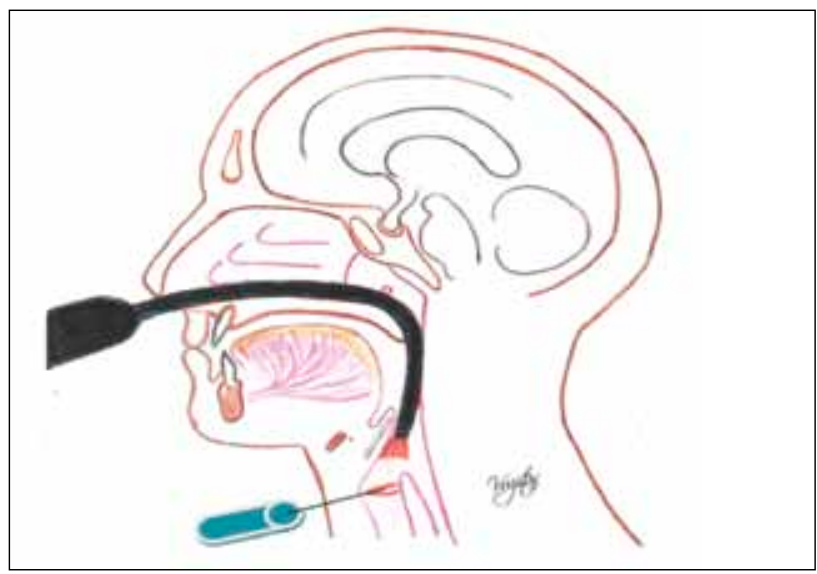

Figure 1 The electrode placement under endoscopic control.

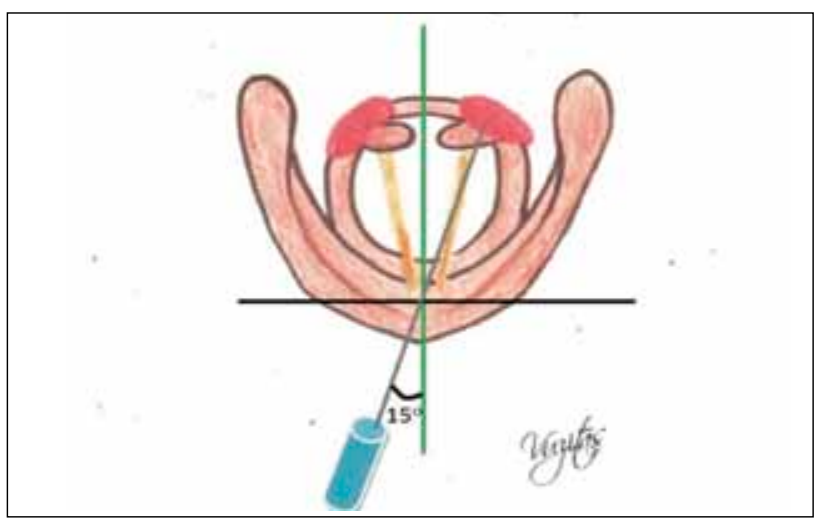

Figure 3 Transcutaneous approach of the posterior cricoarytenoid muscle.
Transcutaneous approach of the thyroarytenoid muscle (Figure 2) involves that the needle pierces through the skin in the sagittal plane, in direct contact with the lower border of the thyroid cartilage, then the needle tip is angled laterally $\left(30^{\circ}\right)$ and superiorly, and penetrates through the cricothyroid ligament (Figure 2).

The correct placement of the needle electrode can be highlighted by an increase in the strength of voluntary muscle contraction and EMG activity while the patient is phonating ${ }^{11}$.

For the posterior cricoarytenoid muscle is the same technique, except that the needle will be angled laterally and superiorly $\left(15^{\circ}\right.$ ) (Figure 3).

The cricothyroid muscle is a superficial muscle which makes it quite approachable. In this case, it is the same technique described above, except that the needle pierces through the skin $5 \mathrm{~mm}$ laterally of the anterior cervical midline and it is angled laterally $\left(50^{\circ}\right)$, penetrating tangentially the cricothyroid membrane (Figure 4).

LEMG is a minimally invasive technique that may cause to the patient: discomfort, cough and painful swallowing, when the needle electrode is at the upper airways and the space tightness. Another incident that may occur is the risk of local infection; systemic antibiotics can be administered after the intervention if there are signs of infection ${ }^{8}$.

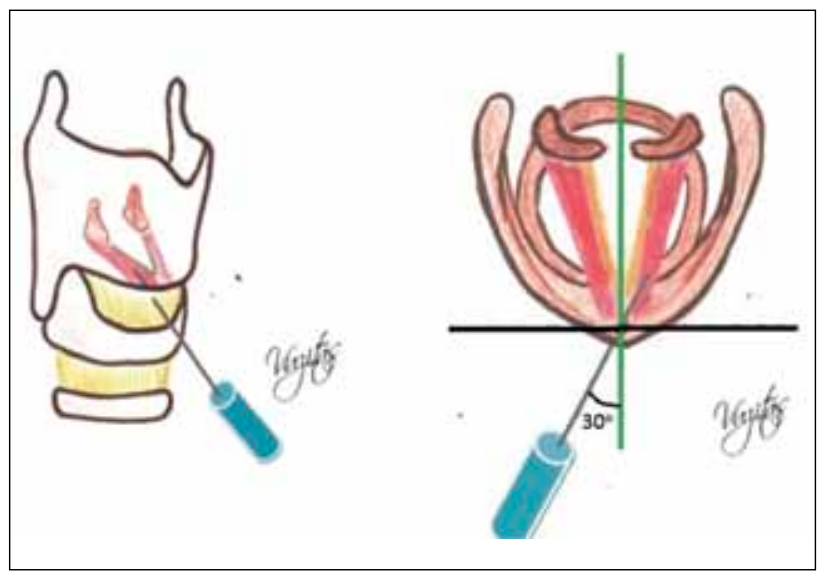

Figure 2 Transcutaneous approach of the thyroarytenoid muscle.



Figure 4 Transcutaneous approach of the cricothyroid muscle. 


\section{LEMG MONITORING}

In 2011, the Working Group on Neurolaryngology of the European Laryngological Society has elaborated a guideline for the interpretation of LEMG. The protocol of EMG examination is standardized and widely used ${ }^{8}$.

The EMG recordings are interpreted by the laryngologist in collaboration with the electrophysiologist. In general, the protocol includes four main parameters of the EMG signal: 1) insertional activity; 2) spontaneous activity; 3) recruitment; 4) morphology of waveform: amplitude of the motor unit action potential (MUAP), MUAP duration and MUAP shape.

It is difficult to assess the voluntary activation of a single laryngeal muscle because during the movement of the vocal cords always participate more laryngeal muscles. There are some tricks that we can use in order to highlight the correct insertion of the electrode. The patient is asked to phonate /i:/, to breathe deeply, to hold his breath in a glottal stop or to swallow. The correct position of the needle is confirmed by detecting increased EMG activity during these physiological reactions 8 .

1. Insertional activity. The insertional activity is characterized by the electrical signal resulting from the insertion of a needle electrode into a muscle. During the quick penetration of the needle through the muscle, muscle fibers are depolarized in a brief burst. Usually, it appears as a positive or negative high frequency spike in a cluster and it is accompanied by a crisp static sound over the loudspeaker.

The insertion activity was categorized as: no activity, normal (Figure 5), decreased, increased or highly increased activity.

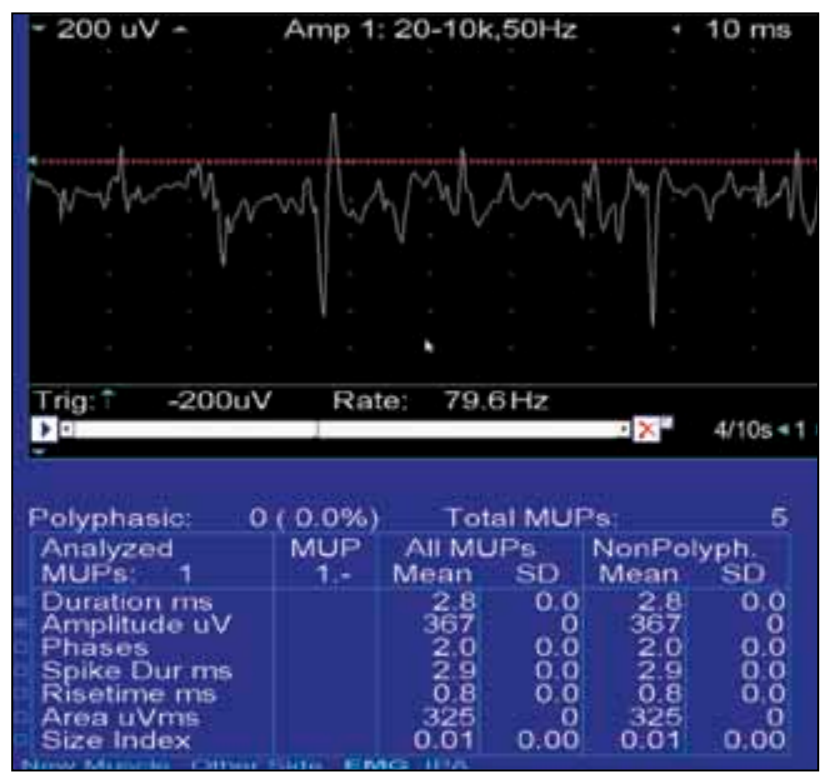

Figure 5 EMG recording during a normal insertional activity of the right cricothyroid muscle - normal MUAP (property of the Functional Explorations Laboratory "CESITO Centre", "Sfanta Maria" Hospital)
Normally, the duration of the insertional activity is less than 300 msec.

Increased insertional activity may be caused by nerve or muscle injuries: neuropathic and myopathic conditions. During the healing process of the nerves or muscles, the normal tissue can be replaced with scar tissue or fat, the result being a decrease in insertion activity.

2. Volitional activity is represented by the recruitment, which is the second parameter evaluated on the LEMG recording. Recruitment pattern may be defined as the successive activation of the same motor units with increasing strength of voluntary muscle contraction.

Normal recruitment pattern: During a minimal voluntary muscle contraction, a single motor unit is seen firing at 2-5 Hz. The average duration of the MUAP from the laryngeal muscle between 2 discharges is of approximately 5 to 6 milliseconds (ms), corresponding to a firing rate of 200 to 500 microvolts.

Recruitment with maximal volitional effort: With increasing effort, the firing rate of individual MUAP increases and progressively new and larger units are recruited and the action potentials of single motor units cannot be revealed from another. The recruitment pattern with maximal volitional effort is equivalent to the "interference pattern".

The incomplete interference pattern of the laryngeal muscle may be classified as follows:

1. No activity.

2. Single-fiber pattern (single unit pattern).

3. Mildly decreased recruitment pattern (incomplete interference pattern).

4. Moderately decreased recruitment pattern (reduced interference pattern). It is typically identified in patients with neurogenic pathology, amyotrophic lateral sclerosis, polymyositis or muscular dystrophies.

5. Normal/dense recruitment pattern - a "full" or "complete" interference pattern is present in a healthy patient during a maximal volitional effort (Figure 6).

3. Pathological spontaneous activity is a parameter categorized as absent (usually observed in normal muscle tissues) or present (little/ moderate/ dense pathologic spontaneous activity). The presence of spontaneous activity can be characterized by the identification of fibrillations, positive waves and high frequency complexes.

The electrophysiologic records of pathological spontaneous activity are classified as following: neurapraxia, axonotmesis or neurotmesis ${ }^{11}$. Absence of spontaneous activity is specific to neuropraxia (Figure 7). Neuropraxia is characterized by a rarefied recruitment pattern without fibrillation activity, without spontaneous positive sharp waves, and the absence of spontaneous activity at rest. 




Figure 6 EMG recording of the left lateral cricoarytenoid muscle during phonation (recruitment) - normal/dense recruitment pattern, normal biphasic MUAP in a patient diagnosed with arytenoid dislocation. (property of the Functional Explorations Laboratory "CESITO Centre", "Sfanta Maria" Hospital)



Figure 7 EMG recording of the left cricothyroid muscle in a patient with posttracheal intubation dysphonia - neuropraxia = absence of spontaneous activity, normal amplitude and duration, normal latency time. (property of the Functional Explorations Laboratory "CESITO Centre", "Sfanta Maria" Hospital)
Axonotmesis represents a partial disruption of the nerve cell axon, with Wallerian degeneration usually located proximal to the site of the injury ${ }^{11}$ (Figure 8). The prognosis is usually with a poor chance of recovery to a functional level. The rate of effect of regenerating nerve fibers depends on the distance involved from the site of injury. The electrophyological parameters describing the axonotmesis are: dense pathologic spontaneous activity - fibrillations, recruitment single fiber pattern, absence of insertion activity.

Neurotmesis is the most severe case of nerve injury and it is characterized by a complete destruction of the entire nerve fiber: axon, myelin sheath, schwann cells and the endoneurium.

a) Fibrillation potentials are characterized as low amplitude potentials (10 to $200 \mu \mathrm{V})$, short duration (1 to $2 \mathrm{~ms}$ ), caused by pathological spontaneous activity of a single-fiber muscle action potentials (Figure 9). These

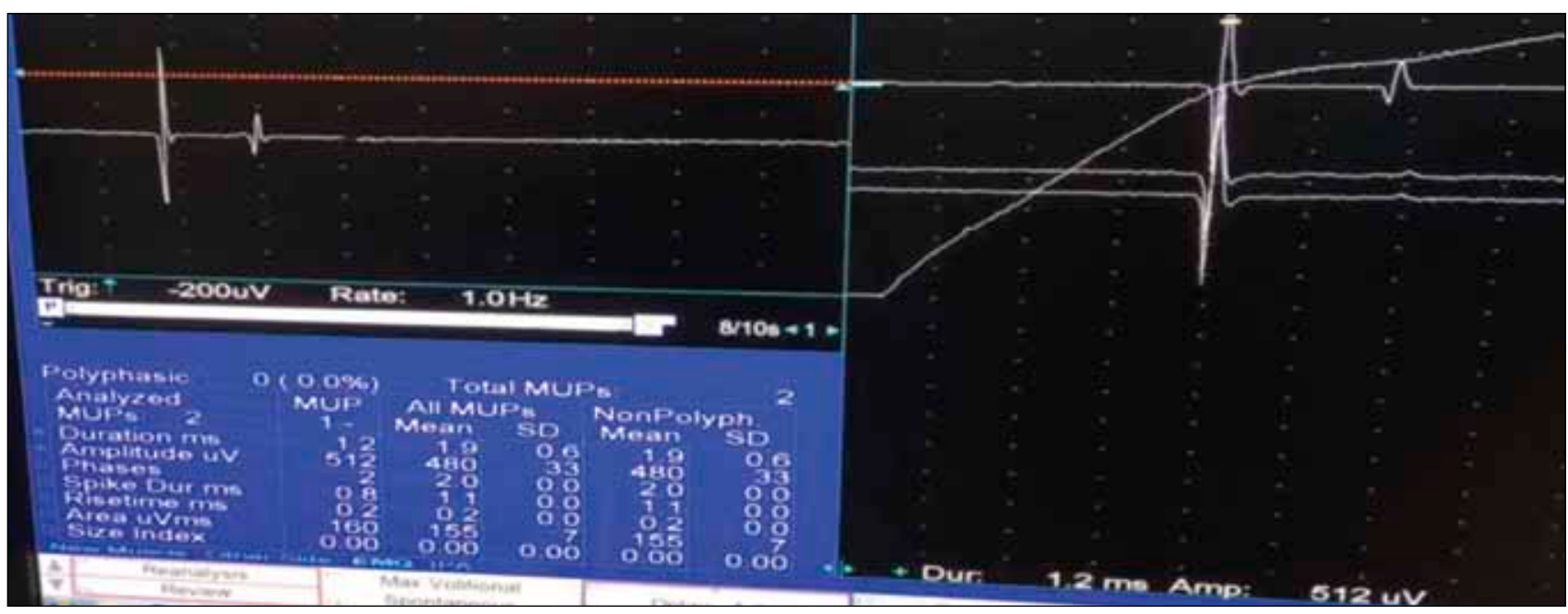

Figure 8 Severe denervation pattern of the left thyroarytenoid muscle (fibrillations) - axonotmesis in a patient with left recurrent nerve injury at about 2 days postthyoidectomy. Presence of fibrillations indicates a poor prognosis of recovery. (property of the Functional Explorations Laboratory "CESITO Centre", "Sfanta Maria" Hospital) 
potentials occur spontaneously with insertion of the needle into the laryngeal muscle; morphology of the waveform is biphasic or triphasic ${ }^{7}$. The presence of a dense pathologic spontaneous activity (fibrillations) after 10 days from a nerve injury indicate a severe degree of denervation and also a unfavourable prognosis.

b) Positive sharp waves (PSW)

Specifically, PSW are defined as low-amplitude (several hundred microvolts), short-duration (1 to $2 \mathrm{~ms}$ ) units generated by a single-muscle fiber indicating axonal degeneration, absence of insertion activity ${ }^{12}$. The feature morphology of the waveform is represented by a large positive deflection, followed by a negative one ${ }^{7}$ (Figure 10).

Fibrillations and PSW have been observed at 2 to 3 weeks after injury and they produce a very specific sound in the loudspeaker of the EMG device.

\section{Morphology of waveform}

Waveform morphology is defined by the presence of the following characteristics: duration, amplitude and the shape of a motor unit action potential (MUAP).

The normal laryngeal motor unit action potential is characterized by: short duration ( 5 - 6ms), small amplitude $(200-500 \mu \mathrm{V})$ and a biphasic waveform which consists of an upward positive spike and a downward negative spike (Figure 5).

The duration of the MUAP is influenced by both the velocity of the neural input and the insulation of the nerve. MUAP amplitude represents the number and the resistance of the muscle fibers innervated by one nerve, nearest to the needle. EMG signals of smaller muscles, particularly of the laryngeal muscles, have a shorter duration and smaller amplitude than the skeletal muscles because they have a high innervation ${ }^{13}$.

MUAP shape means changes in the electrical activity of the muscle membrane. When the needle is in the

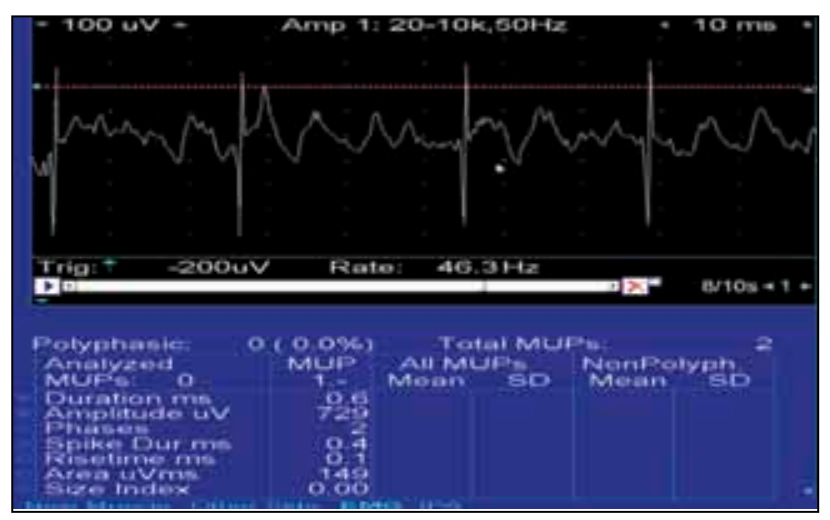

Figure 9 Moderate denervation pattern of the left thyroarytenoid muscle - fibrillations - little pathologic spontaneous activity, minimal recruitment on voluntary action, short duration $(0.6 \mathrm{~ms})$. (property of the Functional Explorations Laboratory “CESITO Centre", "Sfanta Maria” Hospital) proximity of the motor unit, the MUAP changes and turns into "sharp"13. The major spike represents the highest frequency parameter of the motor unit and it has a biphasic pattern with the largest positive-to-negative component of the MUAP; frequently, it is present after the first positive peak. The waveform morphology of the MUAP can give information regarding the recovery ${ }^{13}$. After the nerve injury, two consecutive processes can occur: denervation followed by regeneration. Abnormal motor unit potential morphologies are revealed during the process of regeneration.

The morphology of waveforms may be classified as follows:

1. Normal motor unit potential (biphasic).

2. Early (sometimes polyphasic) reinnervation potentials imply the presence of a recent nerve injury. Electrical signals on the LEMG recordings reveal motor unit potentials with low amplitude, long duration and polyphasic shapes.

3. Giant polyphasic reinnervation potentials are described as highly polyphasic MUAP, with high amplitude and long duration. The MUAP appear as a result of this on-going regeneration. These polyphasic potentials occur at 4 months after the injury (Figure 11).

4. Myogenic polyphasic potentials are produced when the nerve is unimpaired but the muscle is damaged. They are characterized by: decreased amplitude, which is equivalent to decreased muscle weakness, and normal duration by the fact that the nerve is intact.

\section{OUR INITIAL EXPERIENCE}

Since October 2015, we have started performing LEMG on a group of 20 patients diagnosed with vocal fold immobility in the ENT\&HNS Department of "Sfanta Maria" Hospital in Bucharest - the Center of

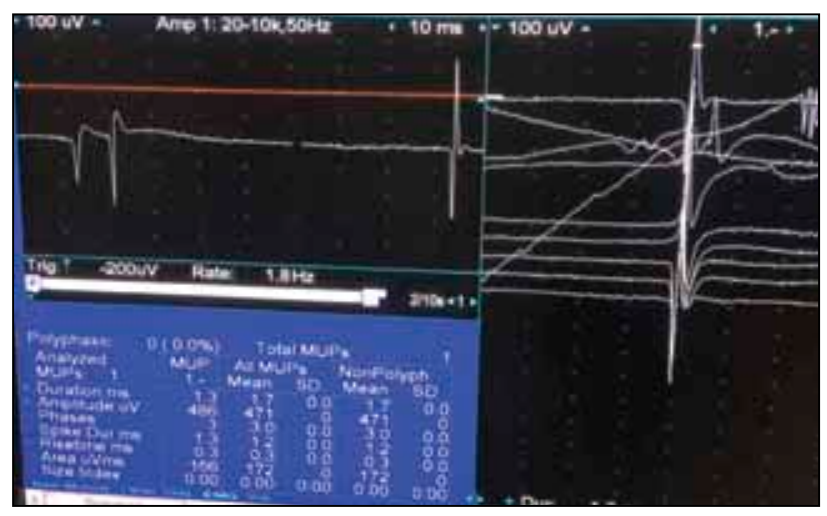

Figure 10 EMG recording - severe denervation pattern of the right thyroarytenoid muscle (fibrillation potentials and positive sharp waves) in a patient with acute injury of the right recurrent nerve. (property of the Functional Explorations Laboratory "CESITO Centre", "Sfanta Maria” Hospital) 


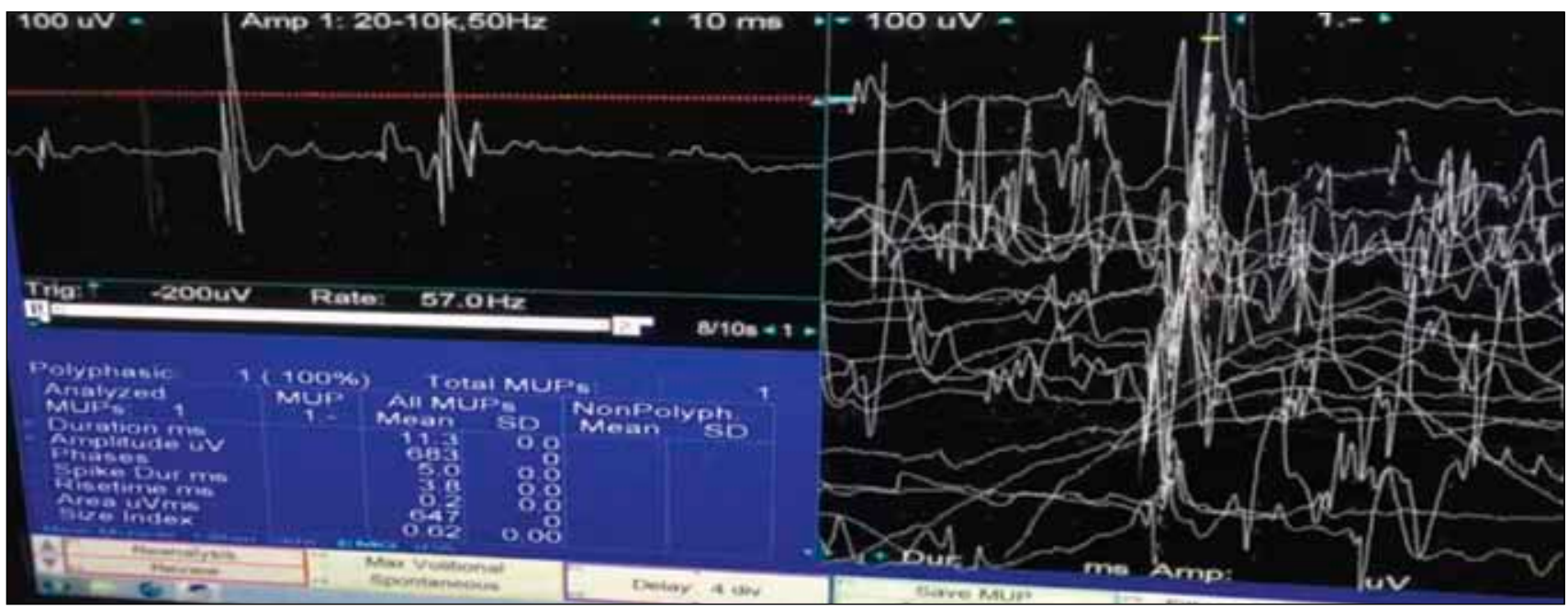

Figure 11 Polyphasic MUAP of right cricothyroid muscle in a patient with right superior laryngeal nerve injury after cervical trauma - giant polyphasic reinnervation potentials: high-amplitude, decreased recruitment, long duration (11ms). (property of the Functional Explorations Laboratory "CESITO Centre", "Sfanta Maria" Hospital)

Excellence for Research of Sensorial and Sensitive Disorders, Study of Infecto-inflammatory, Tumoral and Obstructive Aero-digestive Pathology (CESITO) which was founded due to an European funding programme contest. In our clinic, we assess the vocal fold paralysis or paresis due to the recurrent laryngeal nerve and superior laryngeal nerve lesion using the electrophysiological system NATUS VIKING SYSTEM: EMG/EP 8 channels Software Viking EDX. All LEMG recordings were performed by the same ENT physician in the Functional Explorations Laboratory "CESITO Centre".

In order to obtain a fair and accurate electrodiagnosis, we have collaborated with a neurologist / electrophysiologist from the Neurological Department of "Elias" University Emergency Hospital. This technique requires the patient's capability and willingness to collaborate and actively participate into this assessment procedure.

In our center, we regularly record the electrical activity of the TA muscle and the CT muscle.

In our patients group, 18 patients $(90 \%)$ were found to have evidence of neuropathy on laryngeal electromyography (vocal fold paresis). Thyroid surgery followed by cervical trauma, viral neuritis, cervical trauma and idiopathic lesions accounted for the majority causes of vocal fold paresis or paralysis. The clinical picture indicated the following probable origins for the vocal fold paresis: thyroid surgery $(7 / 18$ or $38.89 \%)$, idiopathic $(4 / 18$ or $22.22 \%)$, viral neuritis $(4 / 18$ or $22.22 \%)$ and cervical trauma $(3 / 18$ or $16.67 \%)$.

We observed that in two patients the TA muscle was difficult to identify during LEMG and in one single patient the CT muscle was not found.

The electromyographic recordings of all 20 patients revealed electrophysiological alterations in 18 of them: presence of fibrillations and positive sharp waves. In two patients we found normal insertional activity with normal motor unit action potential, normal recruitment pattern and absence of spontaneous activity. Corroborating those two patients' data, clinical examination and electromyography, we established the diagnosis of arytenoid dislocation.

The electrophysiologic records helped us identify the nervous site of the lesion: 14 patients $(77.78 \%)$ had a dysfunction of the recurrent laryngeal nerve and 4 patients $(22.22 \%)$ had a dysfunction of the superior laryngeal nerve.

The electrophysiologic findings of denervation were found in all 17 patients diagnosed with unilateral vocal cord paralysis and especially in a single patient diagnosed with bilateral vocal cord paralysis. We found normal EMG recordings in both patients diagnosed with arytenoid dislocation. Early differential diagnosis of joint fixation cricoarytenoid or arytenoids dislocation is essential for further surgical management and satisfactory prognosis ${ }^{12,14}$.

An interesting ascertainment in this study was that in a high number of cases (14 patients) the electrophysiological diagnosis was an on-going axonal loss and in a low number of cases (4 patients) reinnervation waves were present.

It was also important to use an easier method of electrode insertion and verification of the electrode placement under endoscopic control. The electrode placement is easier if the manoeuvre is realised under endoscopic control especially if the patient has a tracheotomy tube. In a patient with tracheostomy the anatomic landmarks are modified because of the presence of fibrosis around the tracheal stoma ${ }^{15}$. We analysed a case of a patient with bilateral recurrent laryngeal nerve injury. In this case, LEMG of the vocalis 
muscle should be performed only on one side at a time, not to cause an exacerbation of the respiratory failure. Fibrilations and polyphasic potentials may be difficult to detect in case of a patient with tracheostomy (Figure 12).

This on-going study will be performed during a 4-year-period and the patients diagnosed with vocal fold immobility will be evaluated in dynamics. The final results will be communicated in the following articles.

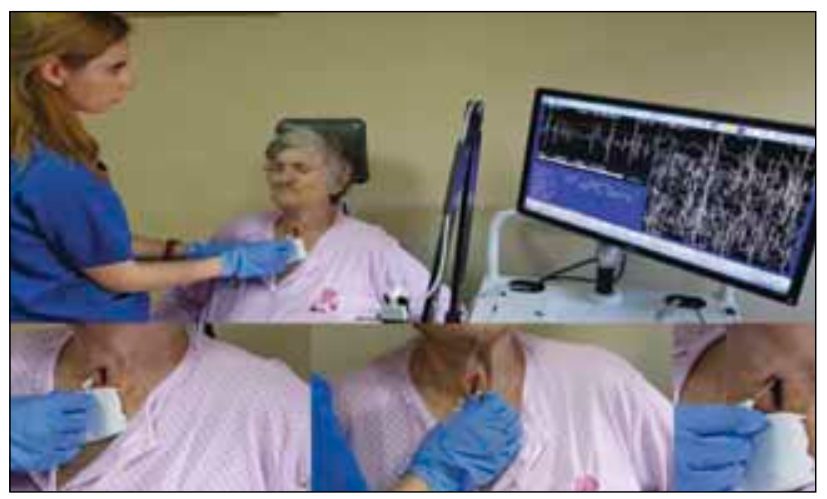

Figure 12 The electrode placement in a patient with tracheostomy. (property of the Functional Explorations Laboratory "CESITO Centre", "Sfanta Maria" Hospital)

\section{CONCLUSIONS}

For the first time, an objective assessment of the laryngeal paralysis can be achieved in the ENT\&HNS Department of "Sfanta Maria" Hospital. In this moment, our clinic is the only one that performs LEMG in Romania.

It is evident that laryngeal electromyography is an indispensable tool in the neurolaryngology requiring a multidisciplinary approach and it is a safe method for assessing vocal fold immobility, especially vocal fold paralysis. Laryngeal electromyography records are useful for identifying the site of the recurrent and superior laryngeal nerve injury.

Interpretation of clinical electrophysiology is a fundamental part in neurology training, but not in otorhinolaryngology, which explains the low number of neurophysiologists involved in laryngeal pathology. In our country, it is intended to implement the laryngeal electromyography in all ENT Departments. To acquire this, besides advanced equipment, the investment of time, training and education it is necessary also.

\section{Conflicts of interests: None}

Contribution of authors: All authors have equally contributed to this work.

Acknowledgment: This research has been conducted successfully and realized in the hospital thanks to the acquisition of modern state-of-the-art medical equipment, especially for this project co-financed through the European Fund for Regional Development.

\section{REFERENCES}

1. De Biase N.G., Korn G.P., Gugliemino G., Pontes P. - Laryngeal electromyography in dysphonic patients with incomplete glottic closure. Braz J Otorhinolaryngol., 2012;78(6):7-14.

2. Weddell G., Feinstein B., Pattle R.E. -(1944) The electrical activity of voluntary muscle in man under normal and pathological conditions. Brain: A Journal of Neurology, 1994;67:178-257.

3. Hydman J., Bjorck G., Persson J.K., Zedenius J., Mattsson P. - Diagnosis and prognosis of iatrogenic injury of the recurrent laryngeal nerve. Ann Otol Rhinol Laryngol., 2009;118(7):506-511.

4. Crespo A.N., Kimaid P.A., Machado Junior A.J., Wolf A.E. - Laryngeal Electromyography: Are theResults Reproducible? J Voice, 2015;29(4):498500. doi: 10.1016/j.jvoice.2014.09.009. Epub 2015 Feb 27.

5. http://www.elsoc.org. Accessed August 29, 2016

6. Rickert S.M., Childs L.F., Carey B.T., Murry T., Sulica L. - Laryngeal electromyography for prognosis of vocal fold palsy: a meta-analysis. Laryngoscope, 2012;122(1):158-161.

7. Sataloff R.T., Praneetvatakul P., Heuer R.J., Hawkshaw M.J., HemanAckah Y.D., Schneider S.M., Mandel S. - Laryngeal electromyography: clinical application. J Voice, 2010;24(2):228-234.

8. Volk G.F., Hagen R., Pototschnig C., Friedrich G., Nawka T., Arens C., Mueller A., Foerster G., et al. - Laryngeal electromyography: a proposal for guidelines of the European Laryngological Society. Eur Arch Otorhinolaryngol., 2012;269(10):2227-2245.

9. Heman-Ackah Y.D., Mandel S., Manon-Aspaillat R., Abaza M.M., Sataloff R.T. - Laryngeal electromyography. Otolaryngol Clin North Am., 2007;40(5):1003-1023.

10. Storck C., Gehrer R., Hofer M., Neumayer B., Stollberger R., Schumacher R., Gugatschka M., Friedrich G., Wolfensberger M. - Laryngeal electromyography: electrode guidance based on 3-dimensional magnetic resonance tomography images of the larynx. J Voice, 2012;26(1):110-116,

11. Sittel C., Stennert E., Thumfart W.F., Dapunt U., Eckel H.E. - Prognostic value of laryngeal electromyography in vocal fold paralysis. Arch Otolaryngol Head Neck Surg., 2001;127(2):155-160.

12. Kimaid P.A., Crespo A.N., Quagliato E.M., Wolf A., Viana M.A., Resende L.A. - Laryngeal electromyography: contribution to vocal fold immobility diagnosis. Electromyogr Clin Neurophysiol., 2004;44(6):371—414.

13. Preston D.C., Shapiro B.E. - Electromyography and Neuromuscular Disorders. Clinical-Electrophysiologic Correlations 2nd Edition. Elsevier, 1998;pp.215-230.

14. Ysunza A., Landeros L., Pamplona M.C., Prado H., Arrieta J., Fajardo G. - The role of laryngeal electromyography in thediagnosis of vocal fold immobility in children. Int J Pediatr Otorhinolaryngol., 2007;71:949-958.

15. Berkowitz R.G. - Natural history of tracheostomy-dependent idiopathic congenital bilateral vocal fold paralysis. Otolaryngol Head Neck Surg., 2007;136(4):649-652. 